\title{
Patterns in the amount of discharge at split-thickness skin graft donor sites
}

\section{Jun-Ho Lee, Chan-Su Kang, Tae-Gon Kim, Kyu Jin Chung}

Department of Plastic and Reconstructive Surgery, Yeungnam University College of Medicine, Daegu, Korea
Background Split-thickness skin grafts (STSGs) are commonly used in the reconstruction of skin defects induced by trauma or burns. Although STSGs are used to successfully treat various wound types, donor site wounds can be challenging, and complications often develop. Therefore, optimal donor site dressings are required. In this study, we introduce an evidence base for patterns in how the discharge amount decreases over time, with the goal of providing insights into the selection of dressing materials. Methods Twenty patients with burns who received STSGs harvested from the thigh between January 2016 to April 2017 were prospectively reviewed. A donor site dressing with foam was changed daily. The weight of the foam dressing was measured before and after placement on the donor site. The wound area was calculated using Visi-Trak. The mean weight of the discharge amount per unit area $\left(\mathrm{g} / 10 \mathrm{~cm}^{2}\right)$ was calculated.

Results The mean weight of the discharge amount per unit area $\left(\mathrm{g} / 10 \mathrm{~cm}^{2}\right)$ decreased from 3.84 to $2.02(P<0.05)$ and $1.09(P<0.05)$ on postoperative days 5 and 10 , respectively. It further decreased to 0.61 by postoperative day 14 .

Conclusions To prevent infections induced by donor site leakage, the use of highly-absorptive foam materials until 5 days after the operation is beneficial. Moreover, hydrocolloid or highly-absorptive foam materials can be used to promote re-epithelialization of the donor site after 5 days postoperatively. For re-epithelialization and wound protection, it is effective to use hydrocolloid materials starting on postoperative day 10.

Keywords Skin grafting / Tissue donor / Exudate

\section{INTRODUCTION}

Split-thickness skin grafts (STSGs) are commonly used in the reconstruction of skin defects induced by trauma or burns [1]. Although STSGs are used to successfully treat various wound types, donor site wounds can be challenging to treat, and complications such as pain, impaired healing, infection, scarring, itching, hy-

Received: Sep 3, 2018 Revised: Sep 20, 2018 Accepted: Sep 20, 2018 Correspondence: Jun-Ho Lee Department of Plastic and Reconstructive Surgery, Yeungnam University College of Medicine, 170 Hyeonchung-ro, Nam-gu, Daegu 42415, Korea

Tel: +82-53-620-3482, Fax: +82-53-626-0705, E-mail: junojunho@gmail.com

Copyright @ 2019 The Korean Society for Aesthetic Plastic Surgery.

This is an Open Access article distributed under the terms of the Creative Commons Attribution Non-Commercial License (http://creativecommons.org/licenses/by-nc/4.0/) which permits unrestricted non-commercial use, distribution, and reproduction in any medium, provided the original work is properly cited. www.e-aaps.org popigmentation, and hyperpigmentation often develop. Impaired healing of the donor site can cause prolonged hospitalization and more problems than the original injury.

Optimal donor site dressings accelerate the healing process by maintaining humid conditions, absorbing excess exudate, and promoting re-epithelialization without the complication of infection [2-5]. Dressings should be impervious to external microorganisms and comfortable for the patient. Moreover, dressings should be soft and flexible to facilitate the identification of irregular wound surfaces. Furthermore, they should be able to adapt to donor sites of various sizes. Existing dressing materials satisfy only some of these criteria. Numerous dressing materials for skin graft donor sites have been discussed. Although numerous dressing materials are applied at donor sites, there is no evidence-based consensus regarding the optimal dressing selection.

In this study, we introduce an evidence base for patterns in how the discharge amount decreases over time for use when selecting 
dressing materials. Moreover, we hope that our study will provide guidance for the selection of appropriate dressing materials for optimal outcomes based on a consideration of the amount of discharge.

\section{METHODS}

This prospective study was conducted from January 2016 to April 2017 and included 20 patients with burns who required STSG. Patients with burn wounds of more than $20 \%$ of the total body surface area, those who abused substances, and those with chronic disease were excluded. All STSGs were harvested in a standardized manner from the anterolateral thigh region with an electrical dermatome (Acculan Dermatome, Aesculap Power Systems; Aesculap Inc., Center Valley, PA, USA) adjusted to a thickness of $0.2 \mathrm{~mm}$. The donor sites were covered with an epinephrine-soaked gauze immediately after harvesting until the end of the operation. After the operation, a 5-mm-thick absorptive foam (Mepilex; Mölnlycke Health Care, Gothenburg, Sweden) was applied. Before applying the absorptive foam, the weight of the foam was measured using an electronic compact scale (SF-400C, GNTECH). An occlusive film dressing (AIDerm Roll; EVERAID Co. Ltd., Yangsan, Korea) was applied over all donor-site foam dressings to avoid leakage or evaporation of the donor site discharge. Cases in which leakage over the foam dressing took place were also excluded. The following day, the skin graft donor-site dressing was changed with a new absorptive foam. At this time, the weight of the foam used on the previous day was measured to calculate the amount of donor site discharge. In this way, dressing changes were carried out every day until the wound and covering dressings displayed no discharge and remained dry (Fig. 1).

When no exudate remained and there was no pain upon expo- sure to air, re-epithelialization was considered complete. Previous studies have not presented a comparison of two sites, which is an example of indiscriminate technique and careless selection of data. Pain was assessed using a scale ranging from 0 (no pain) to 5 (maximally severe pain), and the score was recorded in a blinded manner every day after dressing removal. Pain at the donor sites was rated by the patients themselves. Scarring of the donor sites was estimated in a blinded manner using the Vancouver Scar Scale (VSS) at 60 days after surgery.

The wound area was calculated using Visi-Trak (Smith \& Nephew, North Ryde New South Wales, Australia). The mean weight of the discharge amount per unit area $\left(\mathrm{g} / 10 \mathrm{~cm}^{2}\right)$ was calculated using SPSS version 23.0 (IBM Corp., Armonk, NY, USA). All donor sites with infection and inflammation were excluded.

\section{RESULTS}

A total of 20 patients received an STSG in this study. There were 13 men and seven women, with a mean age of 53 years (range, 25-83 years). The donor site dimensions ranged from 64 to $480 \mathrm{~cm}^{2}$, with a mean of $242 \mathrm{~cm}^{2}$. A 5-mm-thick absorptive foam and occlusive film dressing was applied in all patients.

The mean amount of discharge per donor site area $\left(\mathrm{g} / 10 \mathrm{~cm}^{2}\right)$ was determined by measuring the donor site area and the amount of discharge (Table 1). The mean amount of discharge per donor site area was found to show a statistically significant increase as the area of the donor site increased $(\mathrm{P}<0.001)$.

The average pain score was 1.41 (range, $0-1.95$ ), as calculated from 280 measurements. In all 20 patients, scars were assessed at 60 days postoperatively. The results of the VSS for the donor areas of all patients at follow-up (median value, 5; range, $0-13$ ) showed a significant difference compared to the normal skin color (which
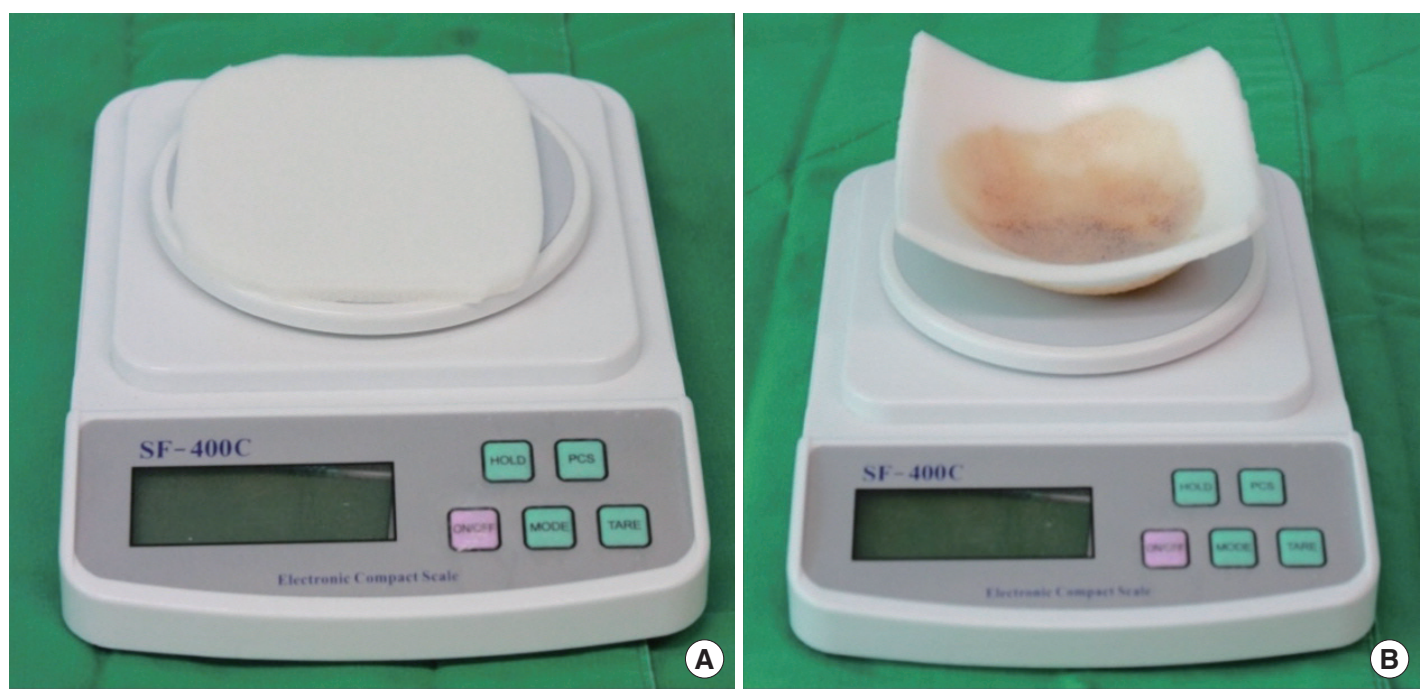

Fig. 1. Electronic compact scale. Weight of the foam before use at the donor site (A) and after use at the donor site (B). 
Table 1. Donor site discharge

\begin{tabular}{lcc}
\hline Patient No. & Mean area $\left(\mathrm{cm}^{2}\right)$ & Mean discharge per area $\left(\mathrm{g} / 10 \mathrm{~cm}^{2}\right)$ \\
\hline 1 & 237 & 4.7 \\
2 & 209 & 4.8 \\
3 & 326 & 5.2 \\
4 & 194 & 4.1 \\
5 & 297 & 5.1 \\
6 & 179 & 3.6 \\
7 & 231 & 4.4 \\
8 & 203 & 6.1 \\
9 & 331 & 5.1 \\
10 & 137 & 3.8 \\
11 & 147 & 3.7 \\
12 & 186 & 3.9 \\
13 & 203 & 4.1 \\
14 & 281 & 4.9 \\
15 & 317 & 5.0 \\
16 & 381 & 5.4 \\
17 & 64 & 2.8 \\
18 & 248 & 4.6 \\
19 & 189 & 4.0 \\
20 & 480 & 7.1 \\
\hline 50 & &
\end{tabular}

$\mathrm{P}<0.001$.

would correspond to a score of 0 by definition).

The amount of discharge at the donor site was highest in the first 2 to 3 days, and it continued to decrease over the course of 2 to 3 weeks. Skin graft donor site discharge continuously decreased $(\mathrm{r}=-0.947, \mathrm{P}<0.0001)$ by about $50 \%(\mathrm{P}<0.05)$ in the first 5 postoperative days and by about $75 \%(\mathrm{P}<0.05)$ by postoperative day 10. Complete re-epithelialization took place by 10 days postoperatively (Fig. 2).

\section{DISCUSSION}

Donor site management should aim to create an environment that allows rapid re-epithelialization, with minimal pain and discomfort. The ideal dressing for STSG donor sites has not yet been identified because of the diverse strategies used to manage donor sites. However, better results in donor sites using occlusive or semi-occlusive moist dressings have been presented in previous studies [613]. Dressings that provide a moist environment, such as hydrocolloids and polyurethane foam, promote re-epithelialization and collagen synthesis [14-16]. In this study, we introduce an evidence base for patterns in how the discharge amount decreases over time for use in selecting optimal dressing materials.

To standardize this prospective study on patterns in the discharge amount, STSG harvesting was performed using the same
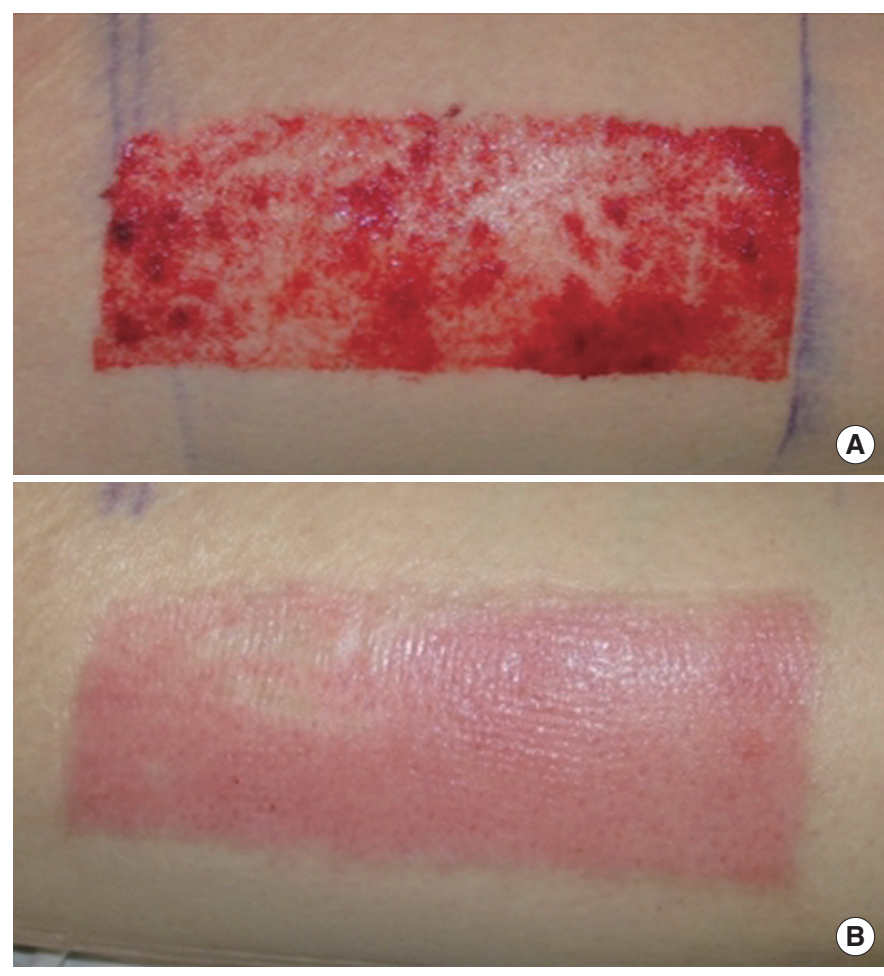

Fig. 2. Donor site of a split-thickness skin graft. (A) Immediate postoperative wound and (B) complete re-epithelization at postoperative day 10 .

protocol and equipment. Dermatome speed and pressure when harvesting the STSG may sometimes influence the final thickness of the STSG. Thus, STSG harvesting was performed by the same surgeon to create fairly uniform donor site wounds.

Pain was assessed using a pain scale. The overall pain scores of patients were low (mean, 1.41; range, 0-1.95). The Veterans Health Administration in the United States has referred to donor site pain as a "fifth vital sign." In other words, donor site pain is one of the most stressful symptoms in the early postoperative period [17]. Thus, a wet environment at the donor sites was created, resulting in less mechanical stimulation. Depending on the definition of complete epithelialization, the time to re-epithelialization may vary. When the definition is based on strict criteria, considering remaining scabs, the time to complete re-epithelialization can be longer. Although we defined complete re-epithelialization as the absence of remaining exudate and the absence of pain to exposed air, there were no remaining scabs on the donor site wounds. The time to complete re-epithelialization was 10 days, which is similar to the results of 10 to 12 days that have been reported in other studies [4,18-21].

The VSS is the most commonly used assessment index for evaluating burn scars [22]. As VSS scores increase, the level of scarring is assessed as worse. We used the VSS to assess the degree of hypertrophic scarring. Although there were differences compared to the 


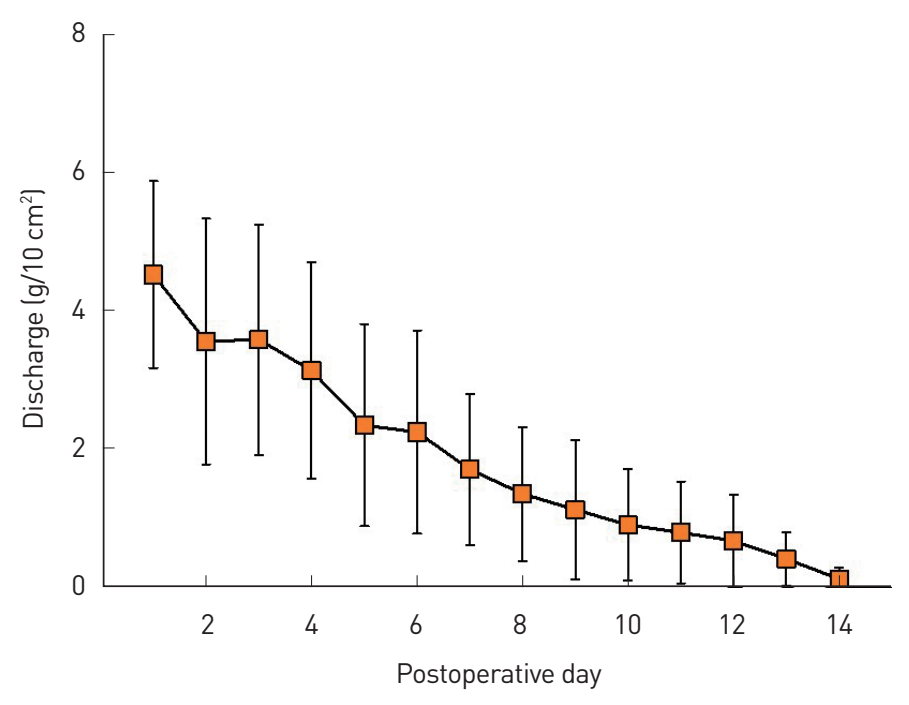

Fig. 3. Mean weight of discharge per unit area $\left(\mathrm{g} / 10 \mathrm{~cm}^{2}\right)$ from postoperative day 1 to postoperative day 14 .

normal skin, the results were acceptable. Moreover, since the period for assessment was relatively short, the results are expected to improve over time. A limitation of this study was that the donor site scar was not a burn scar; therefore, this context was different from the usual criteria for which the VSS is used. In addition, it is necessary to take care when interpreting these results, because the item used to evaluate the degree of pigmentation in the VSS assessment is not an ordered variable.

The amount of discharge at the donor site was highest in the first 1 to 3 days, and it continued to decrease over the course of 2 to 3 weeks (Fig. 3). The fluid handling capacity of the Mepilex was $7.64 \mathrm{~g} / 10 \mathrm{~cm}^{2} / 24 \mathrm{hr}$. This moist wound healing environment was achieved with the foam dressings used in this study. In our prospective study, the amount of discharge decreased markedly throughout 14 days postoperatively, with no cases of infection. A further study using various dressing materials and a longer followup period are needed. Nevertheless, we hope that our study could provide guidance for the selection of appropriate dressing materials for optimal outcomes, considering the discharge amount.

In conclusion, we recommend using highly-absorptive dressing materials until 5 days preoperatively to prevent donor site leakage, which may induce infection, and then using hydrocolloid or highly-absorptive dressing materials to promote re-epithelialization of the donor site after 5 days postoperatively. Subsequently, after 10 days postoperatively, hydrocolloid dressing materials are recommended to facilitate re-epithelialization and protection of the donor site. Based on the findings of this prospective study, tailoring the treatment method based on the amount of exudate after surgery will provide meaningful results.

\section{CONFLICT OF INTEREST}

No potential conflict of interest relevant to this article was reported.

\section{ETHICAL APPROVAL}

The study was performed in accordance with the principles of the Declaration of Helsinki.

\section{PATIENT CONSENT}

The patient provided written informed consent for the publication and the use of the images.

\section{ORCID}

Jun-Ho Lee (https://orcid.org/0000-0002-0062-6420)

Chan-Su Kang (https://orcid.org/0000-0002-2005-6779)

Tae-Gon Kim (https://orcid.org/0000-0002-6738-4630)

Kyu Jin Chung (https://orcid.org/0000-0001-6335-1818)

\section{REFERENCES}

1. Fowler A, Dempsey A. Split-thickness skin graft donor sites. J Wound Care 1998;7:399-402.

2. Feldman DL. Which dressing for split-thickness skin graft donor sites? Ann Plast Surg 1991;27:288-91.

3. Ramirez OM, Granick MS, Futrell JW. Optimal wound healing under Op-Site dressing. Plast Reconstr Surg 1984;73:474-5.

4. Dornseifer U, Fichter AM, Herter F, et al. The ideal split-thickness skin graft donor site dressing: rediscovery of polyurethane film. Ann Plast Surg 2009;63:198-200.

5. Barnea Y, Amir A, Leshem D, et al. Clinical comparative study of aquacel and paraffin gauze dressing for split-skin donor site treatment. Ann Plast Surg 2004;53:132-6.

6. Flanagan M. Outside influences. Nurs Times 1992;88:72-8.

7. Hermans M. An overview of physiological aspects of occlusive and non-occlusive dressings. Prim Intent 1995;3:8-13.

8. Barnett A, Berkowitz RL, Mills R, et al. Comparison of synthetic adhesive moisture vapor permeable and fine mesh gauze dressings for split-thickness skin graft donor sites. Am J Surg 1983;145:379-81.

9. Bilwani PK, Sheth $H$. The use of Lyofoam as a dressing for split skin graft donor sites. Indian J Plast Surg 1990;20:38-40.

10. Iregbulem LM. Use of a semi-permeable membrane dressing in donor sites in Nigerians. Ann Acad Med Singapore 1983;12(2 Suppl):425-9.

11. Sawada Y, Yotsuyanagi T, Sone K. A silicone gel sheet dressing containing an antimicrobial agent for split thickness donor site wounds. Br J Plast Surg 1990;43:88-93.

12. Steenfos HH, Partoft S. Comparison of SureSkin ${ }^{\circledR}$, DuoDerm $E^{\circledR}$ and Jelonet ${ }^{\circledR}$ gauze in split skin donor sites: a clinical and histologial evalu- 
ation. J Eur Acad Dermatol Venereol 1997;8:18-22.

13. Terren J, Serna C, Tejerina C, et al. A comparative study of three new occlusive dressings for healing of graft donor sites versus conventional therapy. Eur J Plast Surg 1993; 16:98-103.

14. Alvarez OM, Mertz PM, Eaglstein WH. The effect of occlusive dressings on collagen synthesis and re-epithelialization in superficial wounds. J Surg Res 1983;35:142-8.

15. Lohsiriwat V, Chuangsuwanich A. Comparison of the ionic silver-containing hydrofiber and paraffin gauze dressing on split-thickness skin graft donor sites. Ann Plast Surg 2009;62:421-2.

16. Argirova M, Hadjiski O, Victorova A. Acticoat versus Allevyn as a splitthickness skin graft donor-site dressing: a prospective comparative study. Ann Plast Surg 2007;59:415-22.

17. Morone NE, Weiner DK. Pain as the fifth vital sign: exposing the vital need for pain education. Clin Ther 2013;35:1728-32.

18. Demirtas Y, Yagmur C, Soylemez F, et al. Management of split-thickness skin graft donor site: a prospective clinical trial for comparison of five different dressing materials. Burns 2010;36:999-1005.

19. Higgins L, Wasiak J, Spinks A, et al. Split-thickness skin graft donor site management: a randomized controlled trial comparing polyurethane with calcium alginate dressings. Int Wound J 2012;9:126-31.

20. Sagray BA, Lalani S, Mehan V. An alternative coverage for split thickness skin graft donor site wounds. J Foot Ankle Surg 2011;50:369-71.

21. Schwarze H, Kuntscher M, Uhlig C, et al. Suprathel, a new skin substitute, in the management of donor sites of split-thickness skin grafts: results of a clinical study. Burns 2007;33:850-4.

22. Baryza MJ, Baryza GA. The Vancouver Scar Scale: an administration tool and its interrater reliability. J Burn Care Rehabil 1995;16:535-8. 